Journal of Computer Sciences 2 (2): 154-159, 2006

ISSN 1549-3636

(c) 2006 Science Publications

\title{
Comparative Performance Analysis of Mobile IPv6 Protocols: Special Reference to Simultaneous Bindings
}

\author{
Shariq Haseeb and Ahmad Faris Ismail \\ Faculty of Engineering, International Islamic University Malaysia, Jalan Gombak, 53100, K.L., Malaysia
}

\begin{abstract}
Mobile IP is the Internet Engineering Task Force (IETF) proposal to cater for All-Internet Protocol (All-IP) mobility. It forms the backbone for next generation Wireless Internet Technology to provide uninterrupted network service while on the move. Our paper conducts a performance study of the various Mobile Internet Protocol version 6 (IPv6) protocols such as Simple Mobile IPv6, Hierarchical Mobile IPv6, Fast handover Mobile IPv6, their combination and Simultaneous Bindings Mobile IPv6. The paper benchmarks the protocol variations against the standard Mobile IPv6 protocol, by studying them under Quality of Service (QoS) parameters. We propose an evaluation model containing 5 mobile nodes and then gradually ( 5 nodes per stage) increasing the mobile nodes to 50 . The proposed network model is then simulated in an open source simulator NS-2. This paper goes further to propose the most suitable variation of the protocol to use and the challenges faced in deployment.
\end{abstract}

Key words: Hierarchical, fast handoff, hybrid, QoS

\section{INTRODUCTION}

The Internet has been ever growing in terms of its services. As new features are included in the Internet, new users are added to exploit them. Majority of these users are now shifting towards mobile computing due to the abundant availability of hotspots around the neighborhood and flexibility of mobile computing. This shift has resulted in a strong channeling towards the use of IP as a common protocol. The future of mobile network sees users being uninterruptedly connected to network while continuously on a move. The IETF working group has proposed Mobile IPv $4^{[1]}$ and Mobile $\mathrm{IPv} 6^{[2]}$ as the main protocol for node mobility.

Mobility of the nodes requires them to detach themselves from one network and attach to another network in order to receive service. This change from one domain to another requires the old IP connections to be terminated and new connections to be established. Mobile $\mathrm{IP}^{[1]}$ describes a solution to this problem and does not require any modifications to the existing routers.

In mobile IP, each mobile node, i.e. laptop, PDA is assigned an address from its home network. While the node is away from its home domain, it obtains a new IP address from the foreign network. The responsibility of the home agent is to intercept any packets for the mobile node and tunnel it to the nodes new location. Thus, requiring the timely movement and registration of mobile node to the new network.

Registration delay becomes the main cause of degradation of performance as the number of mobile nodes in a network increase. The solution proposed to this problem is a hierarchical scheme ${ }^{[3-5]}$ to lower the address configuration signaling through segregating macro mobility from micro mobility.

Another problem is that during the time span (usually very long periods) where a mobile node is shifting its position from one network to another. There cannot be any transfer of traffic, as the mobile node can't be addressed. To solve this problem, a fast handover mechanism ${ }^{[6]}$ has been proposed.

The Hierarchical and Fast handover mechanisms solve much of latency problems. However, there is yet another problem of excessive packet loss. Where, we don't know when exactly will the mobile node move or reach the new access router. In many cases, the packets are transmitted too early or too late and this dilemma results in huge amounts packet loss. This problem will be solved by our proposal of simultaneous bindings.

Mobile IPv6: Mobile IP supports mobility of nodes by providing them at least two addresses: firstly, a home address, which is provided to it by the home agent and it is a fixed address. Secondly, a care-of address, which is provided by a foreign agent and it changes as the node moves to a new network. The components of Mobile IPv6 are shown in Fig. $1^{[7]}$.

A correspondent node can indirectly send packet to the mobile node via the home agent, if it does not find registration information for the mobile in its binding cache. If the registration information is present in the binding cache of the correspondent node, then the packets can be directly routed to the mobile node.

In order to keep the binding values up dated, the mobile nodes periodically inform the home agent and

Corresponding Authors: Shariq Haseeb, Faculty of Engineering, International Islamic University Malaysia, Jalan Gombak, 53100, K.L., Malaysia, Phone: +60163805770 
the correspondent nodes about its new care-of address if and when it changes its location or after the binding lifetime has expired.

Hierarchical mechanism: Mobile IPv6 handles local mobility (mobility within a site) and global mobility (inter-site) in the same fashion. In Mobile IP, the mobile node sends binding update to the home agent and the correspondent nodes each time it changes its point of attachment regardless of the magnitude of the movement. This procedure causes registration delay and packet loss.

Hierarchical scheme of Fig. 2 is implemented with a help of a Mobility Anchor Point (MAP) that separates local and global mobility. Mobile nodes register with the MAP and the MAP intercepts packets on behalf of mobile nodes and redirects it to the mobile nodes. When a mobile node enters a MAP domain, it is given two addresses, regional address (RCoA) and on-link address (LCoA). The mobile node then sends a binding update to the MAP, which binds RCoA and LCoA. In addition mobile node also registers its RCoA with the home agent and external correspondent nodes. Now, if the mobile node moves within the same MAP domain, i.e. within site 1 of Fig. 2, it simply acquires a new LCoA and updates the MAP and local correspondent (red in color) nodes. Since, the external correspondent nodes communicate using the RCoA (which hasn't changed), the mobile node need not update them.

Fast handover mechanism: This is also known as low latency address configuration protocol because it configures the address of the mobile node before it starts to move from its current point of attachment. Fast handover consists of three phases: handover initiation, tunnel establishment and packet forwarding.

The handover initiation is done by the mobile node sending a Router Solicitation Proxy (RtSolPr) message to the Previous Access Router (PAR) indicating that it wishes to perform a fast handover to a new attachment point. The RtSolPr contains the link-layer address of the new point of attachment, which is derived from the New Access Router's (NAR) beacon message. The Mobile Node will receive, in return, a Proxy Router Advertisement (PrRtAdv) message from the PAR indicating the NAR is unknown, known but connected through same address or known and has specified a new prefix that the mobile node should use. Subsequently, the mobile node sends a Fast Binding Update (F-BU) to the PAR using its newly formed CoA. In return the mobile node receives Fast Binding Acknowledgement (F-Back) to indicate successful binding.

Tunneling phase creates a tunnel between NAR and PAR. To perform this, PAR sends a Handover Initiation (HI) message to the NAR. In response, PAR receives Handover Acknowledgement (HAck) from the NAR.
Finally, the packet forwarding phase is performed to smoothen the handoff until subsequent registration by the mobile node to the home agent is completed. The initiation of forwarding of packets between PAR and NAR is based on an 'anticipated timing interval', that is, the network anticipates the time at which the mobile node is likely to handoff. This kind of interval is very difficult to generalize and forwarding too early or too late will result in huge packet losses. After arriving at the new location, mobile node sends a Fast Neighbor Advertisement (F-NA) to initiate the packet flow from NAR to itself.

Fast handover hierarchical mechanism: This is another attempt to further reduce the overall handoff latency from what its predecessors can offer alone. By this combination, the latency due to address configuration and the home/network registration can be reduced. The MAP can then start functioning as the "local home agent" $"$ "[8] therefore, the signaling cost saved is the difference between the round trip time of the $\mathrm{MN}$ to the HA and MN to the MAP.

Simultaneous Bindings: In many wireless networks it is impossible to know exactly when a MN has detached from the wireless link to old Access Router (oAR) and has attached to the one connected to new Access Router (nAR). Therefore determining the time when to start forwarding packets between oAR and nAR is not possible. Certain wireless technologies involve layer-2 messages, which instruct the $\mathrm{MN}$ to handoff immediately or simply identify that the $\mathrm{MN}$ has detached/attached. However even if the ARs could extract this information, there would not be sufficient time for the oAR to detect the MN's detachment and start getting packets tunneled over to nAR before the $\mathrm{MN}$ attaches to nAR. This is because wireless layer-2 handoff times are quite small (ranging from 10's to 100's ms).

A simple solution to this problem is to bi-cast or ncast the packets for a short period of time, from oAR to one or more future locations of the mobile node (i.e. nARs) even before the mobile node actually moves to that location. The $\mathrm{MN}$ is thus able to receive traffic independently of the exact layer- 2 handoff timing during the handoff period.

The operation of this protocol is effectively similar to Fast handoff protocol except that all Fast Binding Updates will have a new Simultaneous Bindings flag set. We would also need to modify mobile nodes so that they can process new fields in the packets. Two BU lifetime values will be returned: $\mathrm{Bi}$ casting lifetime (in the simultaneous bindings sub option)and new CoA lifetime (in the BA option). The new CoA lifetime (placed in the BA option as specified in MIPv6) starts after the Bi casting lifetime ends. Hence, when the bi casting lifetime ends, the $\mathrm{MN}$ will remove this entry 


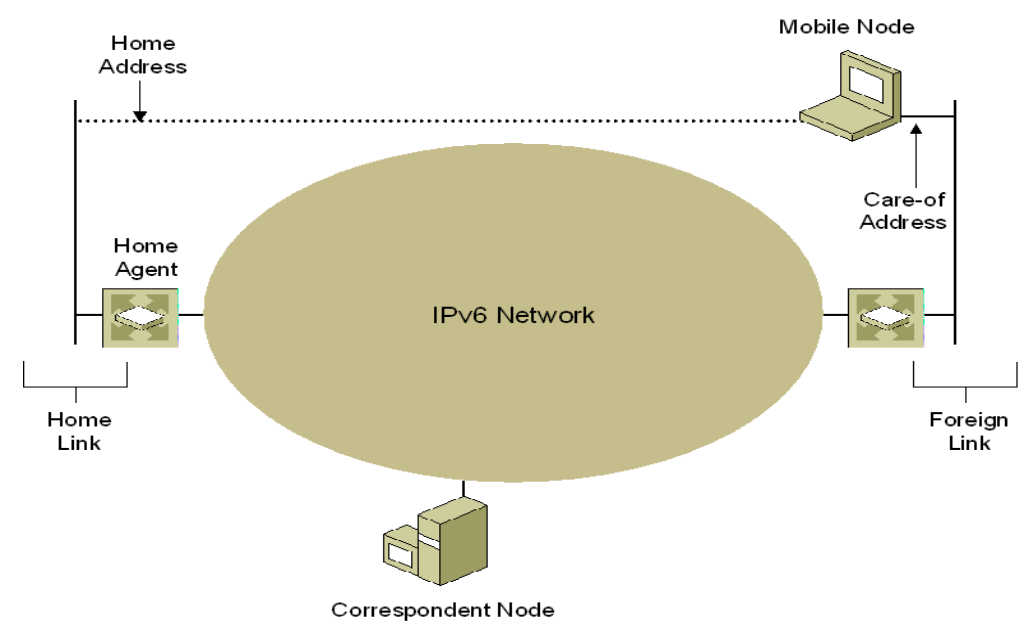

Fig. 1: Basic components of mobile IPv6

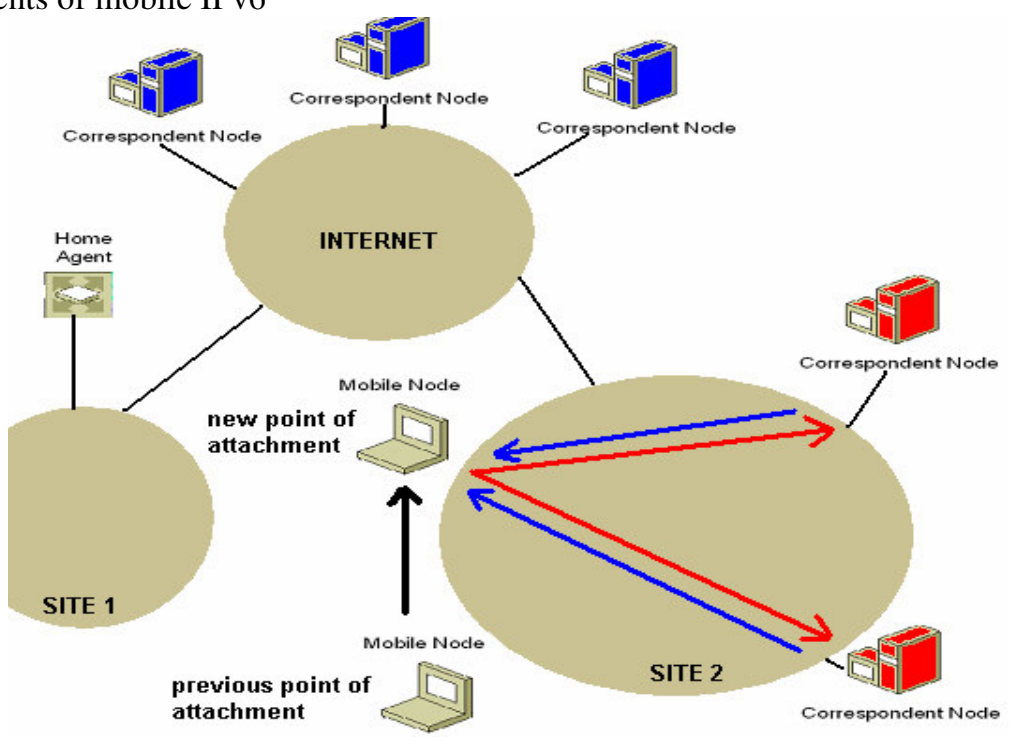

Fig. 2: Intra-site movement of mobile node

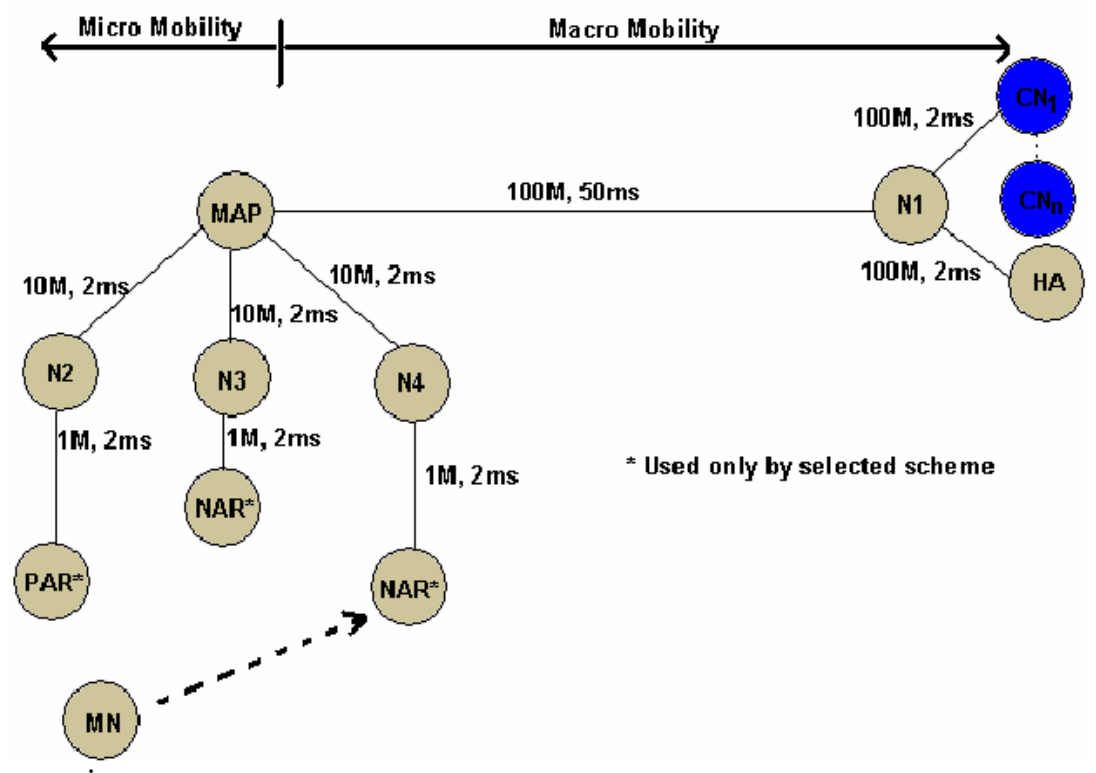

Fig. 3: Simulation scenario 
from the Binding Update list and simply keep one entry for the new CoA with the lifetime returned from the HA/MAP/AR. ${ }^{[4]}$.

In some rare scenarios it may be possible that the MN receives more than one copy of the same packet. Generally the Internet routing mechanisms cannot guarantee a delivery of a single copy of an IP packet to a node. However some TCP congestion avoidance implementations are known to react negatively to the reception of 3 duplicate acknowledgements. These problems can be solved using the algorithms designed to handle multiple copy detection and deletion ${ }^{[4]}$.

IPv6 Deployment Challenges: Mobile IP technology has been standardized but is not implemented in the real world scenario because of the following reasons:

* Lack of usage scenario: This is about the fact that we don't really have an environment to deploy this technology. One scenario that we propose for initiating the usage is the cellular phone network. However, this would incur higher packet overhead unless header compression can be effective. Issues such as communication speed difference and the cost of supporting different technologies makes it unpopular. To solve this problem, a more refined and efficient algorithm must be developed.

* Mobile IPv6 mechanism issue: The mobile IPv6 mechanism requires the routers on foreign links to be IPv6 capable. This poses a problem because it may be very difficult to convince external users to change their systems to support IPv6 traffic, since IPv6 traffic around the world is minimum.

* The IPv6 costs and risks: Mobile IPv6 introduces a privacy risk because it encodes information in the addresses, making this information visible to attackers. One can determine a company's ISP based on the addresses used by the hosts. IPv6 headers can also encode MAC addresses that can reveal the manufacturers of the Ethernet interfaces, which poses a bigger threat. The large IPv6 headers also introduce significant overhead and risk to networks with low bandwidth settings and to those applications that used fixed packet sizes in their protocol operations. Both these problems can be solved by effectively scrambling and compressing the headers so they can't be misused and at the same time are small in size.

* Lack of IPv6 routers: Since IPv4 is still widely in use, routers are making a rapid transition to hardware support for IPv4 wire-speed forwarding, especially for backbone routers. This causes the fear that the IPv6 hardware support will be lagging and expensive. Leading to higher cost for lower performance.
Simulation setup: Keeping in mind the deployment challenges, we propose to study the different variations to find the most suitable algorithm that can be widely accepted. In order to study the performance of different variations of the Mobile IPv6 algorithm, a test scenario was designed. The test scenario was made robust enough to conclude a meaningful result at the same time it had to be simple enough to be simulated within ns- 2 on a limited resourced computer.

The chosen scenario is depicted in Fig. 3. It is composed of a home agent and the correspondent nodes that are connected through node1 (N1, modeling the internet). Mobile node is located in an open space and connected to a distant home agent (HA). The link between node 1 and MAP has a capacity of $100 \mathrm{Mbps}$ with a delay of $50 \mathrm{~ms}$, modeling the distance between the home network (micro mobility) and the current location of the mobile node. Below the MAP is the 'local' network (micro mobility).

In order to model a real world situation, half of the correspondent nodes (depicted in blue) send packets to the mobile nodes, while the other half receive packets from the mobile nodes. The packets are delivered to and from the mobile nodes using the $2 \mathrm{Mbps}$ Wireless LAN $802.11^{[9]}$ provided by ns- 2 .

The traffic sources used for our simulations are UDP CBR source. These provide constant bit rate traffic and require no acknowledgements. This kind of traffic models real time data and is used because of its ease in studying protocol comparisons.

Performance metrics: Performance comparison quantitatively evaluates the variations of the mobile IPv6 protocol variations based on the following parameters:

* Handoff latency: It is the time that elapses between the last packet received and the arrival of the first packet. The higher the handoff latency, the poorer is the performance of a network.

* Packet losses: It is the amount of packet dropped, lost or corrupted during transfer. The higher is the packet loss, the poorer is the performance.

* Bandwidth: The amount of data a node can put on a link is the bandwidth is observes. Higher bandwidth is a symbol of an effective network.

\section{RESULTS AND DISCUSSION}

Figure 4 shows that Simultaneous Bindings Mobile IPv6 (SimMIPv6) has the highest latency because of the amount of signaling load produced by the fast updates and acknowledgements combined with the redundant packet duplications. In comparison we can observe that that the hybrid scheme of HMIPv6 and FMIPv6 gives 
the best result as the number of nodes in crease because it exploits the advantages of both HMIP and FMIP. It is also evident from the result that until 25 nodes, the link delays play an important role in the delays of the algorithms while after 25 nodes, the dominating factor is the algorithm delays.

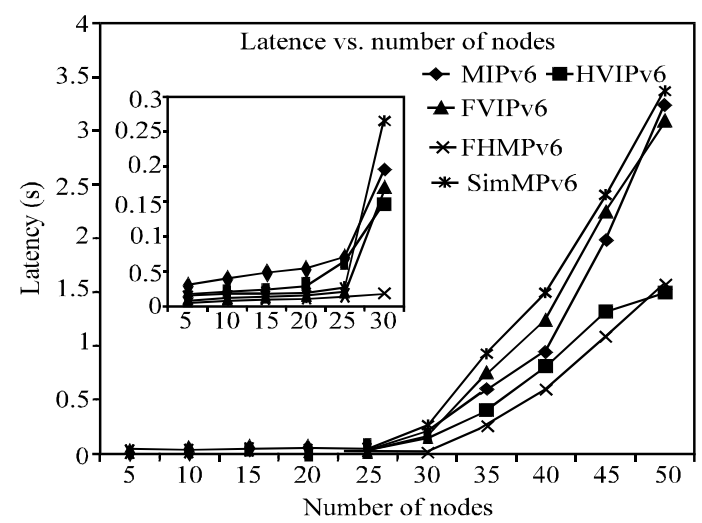

Fig. 4: Effect of number of stations on handoff latency

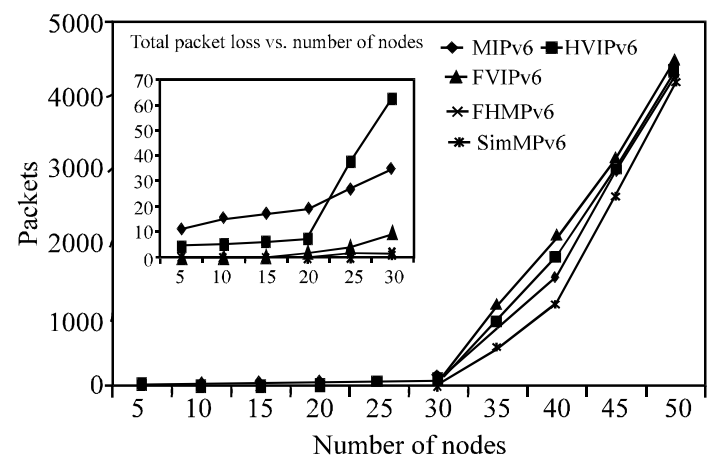

Fig. 5: Effect of number of stations on packet losses at mobile node

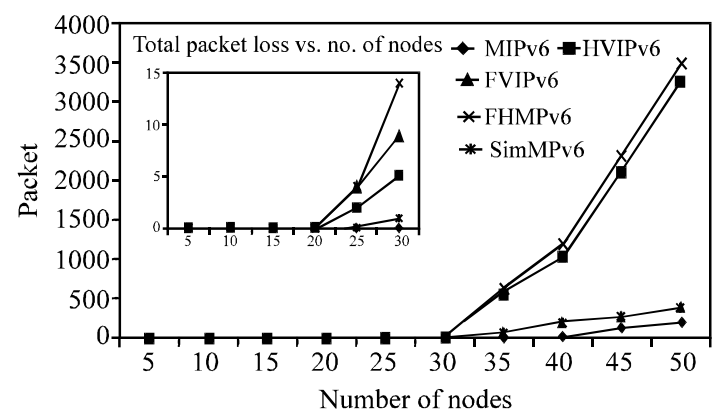

Fig. 6: Effect of number of stations on packet losses at home agent

The effect of handoff latency is also reflected in the results of Fig. 5, where FMIPv6 has the highest number of packet loss. From this result, we can conclude that as the number of nodes increases, the packet drop is the lowest in Simultaneous Bindings Mobile IPv6. This is because SimMIPv6 makes sure that a successful handoff is achieved hence lower packet loss. The tradeoff one has to consider here is that, SimMIPv6

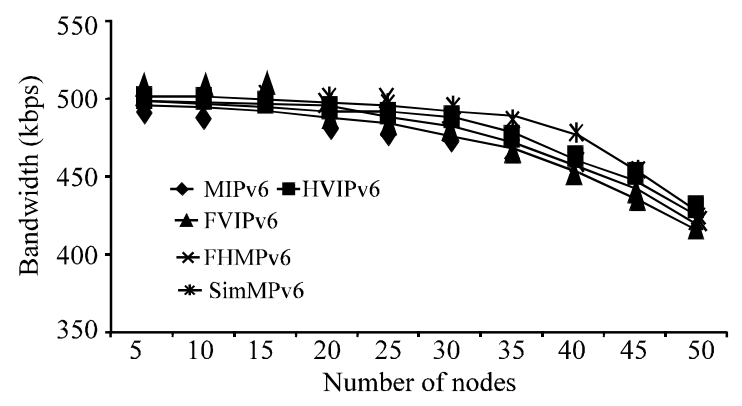

Fig. 7: Bandwidth obtained by mobile node

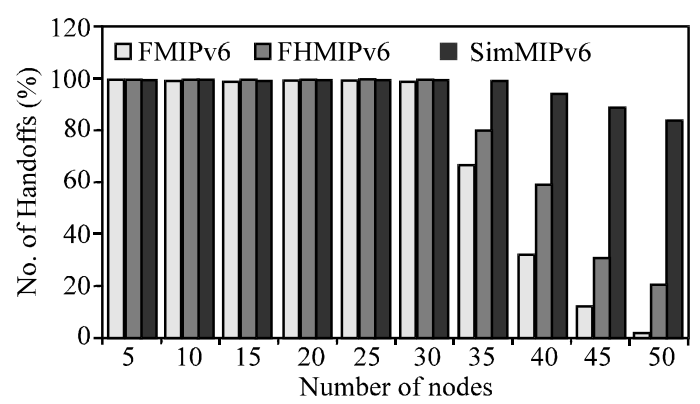

Fig. 8: Number of successful handoffs

gives lower packet loss however the delay is much more which makes it unreliable for real-time traffic.

Figure 6 shows a more conclusive. HMIPv6 and FHMIPv6 present similar packet losses results since the process to update the MAP and afterwards HA and CN about new point of attachment is the same. Packets are lost at HA only when the BU lifetime of both, $\mathrm{CN}$ and HA has expired. The reason for excessive packet loss is that MN has to wait for MAP's BAck to send the BUs to $\mathrm{HA}$ and $\mathrm{CN}$, which can take a long time if the channel is busy.

Figure 7 is in conformity with the results from packet losses. If there is a higher packet loss, the effective bandwidth seen by the $\mathrm{MN}$ is resultantly lower because packet loss is inversely proportional to the throughput.

Figure 8 shows that with the use of SimMipv6, the percentage of total handoffs that are successful increases. This is a very interesting observation because successful handoffs determine the overall performance of the mobile network.

\section{CONCLUSION}

Mobile IPv6 is a key element of the future of AllIP wireless network to allow users to traverse freely between domains and still be connected to a service network. In this paper we have conducted a quantitative analysis of various Mobile IPv6 protocols in development. The results obtained, give conclusive evidence that the hybrid of HMIPv6 and FMIPv6 performs better when we compare the latency that's makes it more suitable for real time traffic. On the other hand SimMIPv6 performs well in terms of packet losses 
and this makes it a suitable algorithm for high and unpredictable traffic applications. We have tested the protocols on our scenario, which can be considered as a close replica of a real world scenario. However, we can only conclude on the efficiency of any of these discussed algorithms after further simulations on a much wider scale.

\section{REFERENCES}

1. C. (Ed.)., 2002. IP Mobility Support for IPv4. RFC 3344.

2. Jonson, D., C. Perkins and J.A. (Eds.)., 2003. Mobility Support in IPv6. Internet Draft. (work in progress).

3. Soliman, H., C. Castelluccia, K. Maliki and L. Bellier, 2002. Hierarchical MIPv6 Mobility Management. Internet Draft, IETF. (work in progress).
4. Maliki, K. and H. Soliman, 2003. Simultaneous Bindings for Mobile IPv6 Fast Handoffs. Internet Draft, IETF. (work in progress).

5. Maliki, K. (Ed.)., 2002. Low Latency Handoffs in Mobile IPv4. Internet Draft, IETF. (work in progress).

6. R., 2002. Performance analysis on Hierarchical Mobile IPv6 with Fast-handoff over End-toEnd TCP. IEEE Global Telecommunications Conference (GLOBECOM).

7. Updated November, 2004. Understanding Mobile IPv6, Microsoft Corporation, Published April 2004.

8. H. J., 2003. Fast Handover for Hierarchical MIPv6 (f-himpv6). Internet Draft. (work in progress).

9. Updated October, 1999. Wireless LAN Medium Access Control (MCA) and Physical Layer (PHY) Specifications. IEEE Standard 802.11. 